(C) 2014 Metaphilosophy LLC and John Wiley \& Sons Ltd METAPHILOSOPHY

Vol. 45, No. 2, April 2014

0026-1068

\title{
BORING INFINITE DESCENT
}

\author{
TUOMAS E. TAHKO
}

\begin{abstract}
In formal ontology, infinite regresses are generally considered a bad sign. One debate where such regresses come into play is the debate about fundamentality. Arguments in favour of some type of fundamentalism are many, but they generally share the idea that infinite chains of ontological dependence must be ruled out. Some motivations for this view are assessed in this article, with the conclusion that such infinite chains may not always be vicious. Indeed, there may even be room for a type of fundamentalism combined with infinite descent as long as this descent is "boring," that is, the same structure repeats ad infinitum. A start is made in the article towards a systematic account of this type of infinite descent. The philosophical prospects and scientific tenability of the account are briefly evaluated using an example from physics.
\end{abstract}

Keywords: fundamentality, fundamental level, grounding, ontological dependence, infinite regress, metaphysical infinitism, gunk, well-foundedness.

\section{What's So Bad About Infinitism?}

There is a widely shared consensus in formal ontology that infinite chains of ontological dependence are vicious. Generally, this is thought to be the case because infinite chains of dependence would have unintuitive consequences. What these consequences are varies according to the type of ontological dependence, but perhaps the most widely discussed case concerns the mereological dependence between a complex object and its parts, that is, its mereological constituents. ${ }^{1}$ The worry is that if a complex object is dependent on its parts, and each part in turn is dependent on its parts ad infinitum, then composition never gets off the ground-we will never reach the fundamental mereological constituents of the object. Recently, several attempts to establish fundamentalism in mereological terms have been made (e.g., Cameron 2008):

Mereological Fundamentality (MF): The world is organized into mereological levels, and the ontological "bottom level" is at one end of the mereological scale.

${ }^{1}$ The term "mereological dependence" is used by Kim, who prefers it to the term "mereological supervenience" and contrasts it with causal dependence: "The properties of a whole, or the fact that a whole instantiates a certain property, may depend on the properties and relations had by its parts" $(2010,183)$. 
MF is generally combined with the idea that there is an asymmetric ontological dependence relation from one end of the scale to the other. It is the direction of this dependence that divides proponents of MF into pluralists and monists. Pluralists typically hold that the direction of the dependence is from the larger to the smaller, resulting in mereological atomism - this is, perhaps, the standard view. Monists think that the parts are dependent on the whole - the whole is prior to its parts - and hence that there is only one fundamental entity, namely, the universe (Schaffer 2010). In what follows, the monist view will be set aside; this article is exclusively concerned with pluralism. The combination of MF and pluralism entails mereological atomism - the view that the ontological bottom level consists of mereological simples. But recently mereological atomism has started to fall out of fashion. ${ }^{2}$ An alternative view is that matter is infinitely divisible atomless gunk (see Schaffer 2010, 61ff.). ${ }^{3}$

The previous worry can now be expressed in terms of gunk: if the world were "gunky" and there were no mereological bottom level, we would never reach the highest degree of reality. More precisely, metaphysical grounding would, as it were, never "bottom out" in something ontologically independent, where something $x$ is ontologically independent if and only if nothing grounds $x$ (Schaffer 2009, 373). Such ontological independents would have the highest degree of reality.

In what follows I use the notions of ground and grounding without further explication. Nothing of substance hangs on the details in this article, so it will simply be assumed that there is a sense of metaphysical explanation captured by these notions. Some authors write about grounding chains rather than chains of ontological dependence, but these notions can be considered interchangeable for the purposes of the present article. In this literature it is often assumed that every non-fundamental truth is ultimately grounded in some fundamental truth.

A related line of thought has been entertained by Ross Cameron (2008), who resists the idea that there could be infinite chains of ontological dependence exactly because of their unintuitive implications. Cameron suggests that "the anti-gunk worry is that composition could never have got off the ground" $(2008,6)$. In other words, if complex objects are ontologically dependent on their mereological parts, then composition never bottoms out in gunky worlds. The intuitive result, according to Cameron, is that complex objects are not possible in gunky worlds. This would rule out the possibility that we live in a gunky world, at least if we accept the plausible idea that complex objects are ontologically dependent

${ }^{2}$ For criticisms of mereological atomism, see, for instance, Markosian 2005 and Ladyman and Ross 2007.

${ }^{3}$ It is not atypical to hold that whatever the mereological structure of the world is, this structure is metaphysically necessary, especially with regard to atomism. However, many recent accounts also question such necessitarianism and hold that gunk is at least possible (e.g., Bohn 2009a). 
on their parts and the common sense intuition that there in fact are composite objects. ${ }^{4}$

If this is correct, then infinite chains of ontological dependence are a very bad sign indeed. For Schaffer, accommodating the possibility of gunk is an important argument in favour of priority monism, but there are other, independent arguments in favour of (the possibility of) gunk (e.g., Sider 1993, Zimmerman 1996). In any case, there is a wide agreement about the incompatibility of MF + pluralism + gunk. ${ }^{5}$ For those of us who are pluralists but take seriously the possibility of the actual world being gunky, this is a worrying result. There appears to be no way to maintain $\mathrm{MF}$ on this view.

A gunky ontology is a type of metaphysical infinitism, which seems to undermine the idea of an ontological bottom level. Metaphysical infinitism, as opposed to metaphysical foundationalism, allows that the (hierarchical) structure of reality could be infinitist, that is, there could be an infinite descent of "levels," each further level dependent on the former in a yet to be specified sense of dependence (cf. Morganti 2009, 272). But does an infinite regress of ontological dependence automatically result in a violation of fundamentality? I contend that this is not the case, at least if we allow for a more liberal sense of "fundamental level," to be specified in what follows.

\section{Infinitism and Well-foundedness}

We have seen that infinite regresses in formal ontology are quite generally considered to be vicious. But we should not conclude that all infinite regresses of ontological dependence are vicious simply because the case of mereological dependence seems to give rise to a vicious regress. One way to proceed here would be to attempt to come up with general criteria for (non-)viciousness. However, the strategy in this article is more modest: it will be suggested that there is at least one sense of infinite regress that does not give rise to viciousness. The idea has been entertained before, if only in passing. For instance, Alexander Paseau suggests that "a definition should not assume that the entities that make up [an entity's] ultimate ontological basis are of finite cardinality, or well orderable, or form a set" (2010, 170; see also Morganti 2009). In the end, however, Paseau also defines the "ultimate ontological basis" in such a way that infinite chains of dependence are ruled out $(2010,174)$.

${ }^{4}$ Cameron does, however, leave open the possibility of gunk, so he thinks that atomism is only contingently true (cf. also Schaffer 2010, 62).

${ }^{5}$ For some counterarguments, see Bohn 2009a and 2009b, and Morganti 2009, which include related discussion concerning "junky" worlds, that is, worlds in which everything is a proper part. Note that this article does not directly engage with "the junk argument" against universal composition proposed by Bohn and discussed in a number of papers since then (see, e.g., Tallant 2013). 
The requirement for ruling out infinite descending chains of dependence in accordance with metaphysical foundationalism is often introduced with the notion of well-foundedness, which is familiar from set theory and the axiom of foundation. We can define well-foundedness for parthood or for some other order. A more or less standard definition is provided in Cotnoir and Bacon 2012: "An order < (in this case proper parthood) on a domain is said to be wellfounded if every nonempty subset of that domain has a $<$-minimal element. We say that $x$ is a $<$-minimal element of a set $S$ if there is no $y$ in $S$ such that $y<x$. Wellfoundedness rules out any infinite descending <-chains. There are atomless mereologies, sometimes called gunky, in which proper parthood chains are all infinite" (Cotnoir and Bacon 2012, 187).

Cotnoir and Bacon are in fact interested in developing a nonwell-founded mereology, which would be able to accommodate gunk. However, we know that Mereological Fundamentality (MF) combined with pluralism is not compatible with such a mereology, due to its association with mereological atomism. This is also clear from Schaffer's analysis (Schaffer 2003, 509-12), as well as from Karen Bennett's (Bennett 2011,34), requiring that a well-founded chain must not be infinite at the fundamental end, even though well-foundedness is compatible with an infinity of grounded entities. Finally, compare this with a recent account of viciousness and infinite regress by Ricki Bliss: "[T]here is an important distinction to be drawn between finite grounding chains and well-founded grounding chains. A finite grounding chain is one which terminates in something fundamental, where we can move from any member of the chain to the fundamentalium in which it terminates in a finite number of steps. A well-founded grounding chain is one that is grounded in something fundamental, but may, itself, be infinitely long. A finite grounding chain is well-founded but a well-founded grounding chain need not be finite" (Bliss 2013, 416).

We can conclude that infinite chains of (mereological) dependence need not be vicious, at least in so far as they are grounded in "something fundamental," that is, they are well-founded.

\section{Boring Infinite Descent}

The specification of the previous section is important, but one should add that an infinite chain that nevertheless terminates does not yet constitute metaphysical infinitism. Such chains are perfectly acceptable to the metaphysical foundationalist. Consider Cameron, for example: "It is false to say that an entity $\mathrm{x}$ is ontologically independent iff there is no entity y such that $\mathrm{x}$ is directly ontologically dependent on $\mathrm{y}$; this thought depends on the assumption that for any dependent entity there is a finite number of steps taking you from it to its ultimate ontological basis. This should be rejected" (Cameron 2008, 4). In other words, the foundationalist only 
needs to insist that the grounding chain eventually terminates, even if it takes infinitely many steps to reach the bottom level. What remains to be settled is in what "fundamental thing" the grounding chain must terminate. In the case of MF, it is of course mereological simples, but there are other alternatives, depending on our conception of reality's hierarchical structure (and hence the type of dependence chain under investigation).

For instance, besides a mereological structure, Schaffer $(2003,500)$ entertains three alternative senses of reality's hierarchical structure, namely, a supervenience structure ordered by asymmetric dependencies, a realization structure ordered by functional relations, and a nomological structure ordered by one-way bridge principles. Some of these alternatives - especially the mereological structure - have been strongly opposed, for example, by Ladyman and Ross (2007, 151). But there is one live option that is of special interest, building on the idea of a supervenience structure and a fundamental "atomless supervenience base."

Schaffer does not provide an explicit definition of the supervenience structure that he has in mind, but the idea can be illustrated by "boring" infinite descent: it's turtles all the way down. There is no novelty in the structure after a certain point. ${ }^{6}$ This means we can follow the mereological chain of dependence all the way to the supposed mereological bottom level, but this is not where the story ends. The whole mereological chain of dependence could supervene on another, infinite chain of dependence (of a non-mereological kind). The boring part of the structure that repeats infinitely could be of any length, as long as it starts anew eventually. A description of the repetitive part only needs to be supplemented with an instruction to continue as before, for example: "The world stands on four elephants, the four elephants stand on a turtle, the turtle stands on two camels, the camels stand on four elephants, the four elephants stand on a turtle ... and repeat ad infinitum." On the face of it, this type of chain does not satisfy well-foundedness, because it is genuinely infinite. But the situation is not as simple as that, for it is now being proposed that the mereological chain terminates, even though it is dependent, in some sense, on another (non-mereological) chain: a two-tiered structure. A simple illustration, sketched in figure 1, might be useful.

In Schaffer's terminology, the mereological chain terminates in a "fundamental supervenience base" $(2003,501)$, more or less in the sense of Humean supervenience. In what follows, I will use a more specific sense of dependence, instead of "supervenience." Nevertheless, it is crucial to make clear that once the boring structure starts, we are done with mereological dependence. So, the chain of mereological dependence as a whole may be

\footnotetext{
${ }^{6}$ The term "boring" in this connection was coined by Schaffer $(2003,505,510)$; "repetitive" might be a better term, especially since this type of infinite descent is in fact rather interesting.
} 


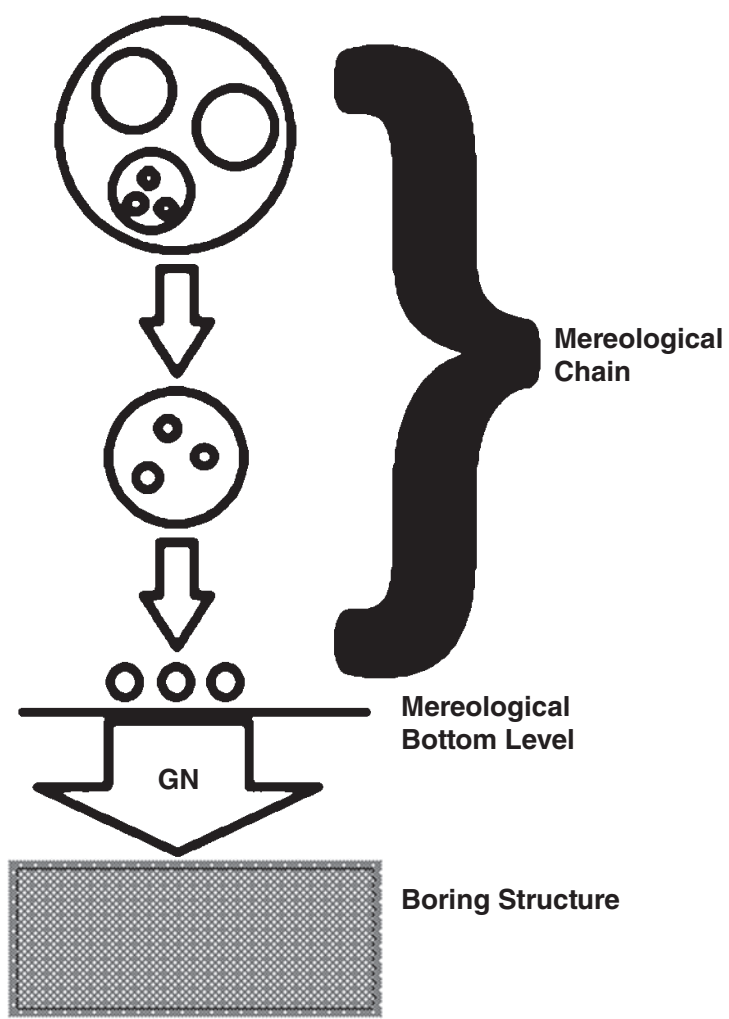

FIGURE 1

considered to depend for its existence on the boring structure; this dependence is illustrated by the GN (generic necessitation) arrow in figure 1. How should we understand the relationship of the mereological chain and the boring structure? In Schaffer's terminology, the mereological chain just "supervenes" on the boring structure. We can be more precise, for it appears that this relationship fulfils commonly accepted criteria for generic ontological necessitation:

Generic Necessitation (GN): $x$ cannot exist unless something is an $F$.

Here $F$ is a general term - that is, $x$ cannot exist unless another object of the type $F$ exists (Correia 2008, 1015). GN refers to an object's existence requiring the existence of an object of a certain sort rather than a specific object (as is the case with rigid necessitation). For instance, any given 
water molecule cannot exist unless hydrogen atoms exist. Or, more precisely, any given water molecule cannot exist unless the physical constraints required for the existence of hydrogen atoms are fulfilled, which in turn requires the possibility of a certain electron configuration, among other things.

We can now apply GN to the case at hand: the mereological chain of dependence (that is, composite objects) cannot exist without the underlying boring structure. In other words, the boring structure is generically necessitated by the mereological structure, as illustrated by the GN arrow in figure 1 . Of course, so far we have not been given any reason to think that this is correct, but we should first clarify the nature of the claim.

Why should we think that the boring structure is repetitive? Well, at least arguably, the infinite descent of a repetitive structure allows for mereological well-foundedness in the sense that the whole mereological chain generically necessitates "something fundamental," namely, an infinitely descending yet repetitive structure. The bottom level is exhausted by the repetition, that is, the repeating structure as a whole constitutes the fundamental level. This is the more liberal sense of "fundamental level" mentioned earlier. To continue with the previous example, no terms other than four elephants, a turtle, and two camels can be introduced that would describe reality more minimally. We can express the idea-call it ontological well-foundedness - in Schaffer's terminology as follows:

Ontological Well-foundedness (OWF): A chain of dependence is said to be ontologically well-founded if it terminates in a fundamental supervenience base.

To clarify, OWF states that a chain of dependence is said to be ontologically well-founded if it terminates in something fundamental or generically necessitates (GN) something fundamental. So, the thought is that boring infinite descent is a viable fundamental supervenience base and hence constitutes "something fundamental."

Is this line of thought compatible with a gunky ontology? In one sense, it clearly is not: if we consider the mereological chain of dependence to terminate, then gunk is ruled out. However, since we have adopted a more liberal sense of "fundamental level," we might also adopt a more liberal sense of gunk, concerning structure rather than proper parthood. The idea here would be that the structure could itself be made up (nonmereologically) of substructures, each of which is made up of further substructures, ad infinitum. I do not aim to settle this matter here (it is perhaps only terminological), but it is clear that the possibility of boring infinite descent opens up a number of interesting avenues regardless of whether we consider it to be genuinely gunky. In either case, boring infinite descent is an example of genuine metaphysical infinitism. 
Schaffer's own description of boring descent has one feature that needs to be discussed: Schaffer suggests that the supervenience relations between all lower (presumably non-mereological) levels "go symmetric" (2003, 511). The idea of symmetric supervenience may seem peculiarthe chain of ontological dependence responsible for the hierarchical structure of reality is generally considered asymmetric. Yet, Schaffer suggests that "the boring parts and their wholes each supervene on the other" $(2003,511)$, contra mereological relations, which are necessarily asymmetric. The idea-and here I am interpreting Schaffer, who does not say much more about the topic - is that we should think of repetitive infinite descent as a symmetric whole. This whole could still be thought to contain directional descent, but the generic necessitation relation becomes symmetric. To give an analogy, consider the carbon fullerene structure, an extremely strong, symmetric carbon structure. ${ }^{7}$ Carbon fullerenes require all the bonds between the carbon atoms to be present in order to gain the properties for which they are known. If we break these bonds, the symmetry is gone and the extreme stability of the molecule is lost. In the case of repetitive, layered descent, the idea is that you cannot take away anything from this whole without the hierarchy collapsing above and below. We get a situation in which, for any two members $x$ and $y$ of the supervenience base, if $x$ is generically necessitated by $y$, then $y$ is generically necessitated by $x$. This is the sense in which boring infinite descent could be understood as a symmetric whole - all layers are necessary for producing the supervenience base for the mereological structure and the composite objects that we are familiar with.

Is this suggestion plausible? Schaffer's own analysis of the matter gives few reasons to accept the idea of symmetric supervenience, but I think that we can make some sense of it following the previous line of thought. Alternatively, we could try abandoning the requirement of symmetric supervenience for repetitive infinite descent and insist that an infinitely repeating structure that satisfies OWF is all we need.

In any case, OWF leaves it open whether we have "full-blown fundamentality" or "supervenience-only fundamentality" (with infinite, boring descent), to use Schaffer's terminology (2003, 512). However, "full-blown infinite descent" is not well-founded in any sense, and we are working with the assumption that only an (ontologically) wellfounded chain of dependence preserves the metaphysical foundationalist's intuition that has been receiving attention in the literature. Do we have any reasons to think that such a chain is possible, not to mention actual?

\footnotetext{
${ }^{7}$ I have in mind the buckminsterfullerene, a spherical fullerene molecule with the formula $\mathrm{C}_{60}$. These molecules are extremely stable.
} 


\section{A Physical Model for Boring Infinite Descent}

It is clear that direct empirical evidence of (boring) infinite descent is not forthcoming. But in so far as we take the idea seriously — not just as a mere exercise in formal ontology - we will eventually have to consider how such structure could be physically realized. One speculative physical model, which may give us some clues, has been proposed by the physicist Hans Dehmelt. Schaffer also mentions this model in passing. Dehmelt (1989) proposes an intriguing quark/lepton substructure based on the model of the triton - the nucleus of hydrogen's radioactive isotope tritium $\left({ }^{3} \mathrm{H}\right)$ : "I propose to extend the triton substructure scheme to an infinite number of layers. Below the four layers listed above [up to subquarks], they contain higher order $\mathrm{d}_{N}$ subquarks, with $N=5 \rightarrow \infty$. In each layer the particles are not identical but resemble each other in the same way as quarks and leptons do, with masses varying as much as a factor $10^{8}$. In an infinite regression to simpler particles of ever increasing mass, they asymptotically approach Dirac point particles" (Dehmelt 1989, 8618).

Up to $N=3$, the level of electrons, Dehmelt's model is motivated by current physics, but is speculative from $N=4$ onwards, where electron substructure is postulated. Importantly, Dehmelt $(1989,8618)$ specifies that the particles in each new layer - as $N$ increases - will be held together by new stronger and shorter-range forces (compare the strong nuclear force), overpowering gravitation. As $N$ approaches infinity, we must also postulate infinitely many and infinitely stronger and shorter-range forces. While this may be relatively unproblematic mathematically, the ontological costs can be problematic, as we will see in a moment. Moreover, Dehmelt's model does not postulate symmetric supervenience relations between the levels at any point, contra Schaffer's requirement for boring descent. Notice also that on the face of it, the model would appear to involve mereological levels all the way down, although Dehmelt does not discuss it in these terms. It is open to interpretation, however, what exactly happens at the point when we reach $N=5 \rightarrow \infty$. One option would be to interpret the mereological chain as terminating at this point and the further chain being boring and satisfying generic necessitation (GN) in the sense discussed earlier.

Another complication of Dehmelt's proposal is that even though the model enables infinite descent, Dehmelt proposes that in the actual world the regress does in fact terminate in what he calls a "cosmon"- the heaviest particle ever to appear in the universe. Proposing an updated version of Lemaitre's "world-atom," Dehmelt assumes that in this universe, the $\mathrm{d}_{N}$ subquarks and hence the regress are only realized up to the cosmon. This leads Dehmelt to a highly speculative proposal about the state of the universe immediately preceding the big bang, consisting of "a tightly bound cosmon/anticosmon pair, the cosmonium world-atom of near zero relativistic mass/energy" (1989, 8619). We can, however, set this aside, 
since we are currently interested only in the possibility of a metaphysically infinitist yet ontologically well-founded model.

What about the ontological cost of introducing new forces at each level? The worry here is that if boring infinite descent requires an infinite number of novel forces, its appeal as an ontologically well-founded, fundamental supervenience base may diminish. In fact, Dehmelt's model will also require the introduction of new exchange particles at each level, which is perhaps even more problematic for OWF. Consider the strong nuclear force: it binds protons and neutrons together in the nucleus, overpowering the electromagnetic repulsion between protons. Like all the fundamental forces, the strong nuclear force is an exchange force, that is, it involves the exchange of one or more particles. At the level of protons and neutrons, the strong nuclear force relies on the exchange of mesons, but it is merely a residual force of the strong force (that is, the colour force) that binds together positively charged quarks. In the latter case, the relevant exchange particles are gluons. A full examination of the strong force would require delving into quantum chromodynamics, but all we are interested in here is the apparent need for independent exchange particle(s) at each level where the force is active (for example, mesons and gluons).

The core of the issue is this: if any physical model with infinite descent requires postulating infinitely many new forces and corresponding exchange particles, can such a model ever be ontologically well-founded? That depends on our understanding of a viable fundamental supervenience base. It is not obvious what kind of criteria Schaffer has in mind when he talks about the fundamental supervenience base, but in the previous section it was proposed that a criterion based on the combination of GN and OWF could be developed, that is, the fundamental supervenience base is something that is generically necessitated by the more familiar mereological structure. It is difficult to understand how an infinite number of forces and exchange particles could satisfy OWF. But we would certainly need more input from physics in order to discuss this in a fruitful manner. In any case, here we see the appeal of symmetric supervenience mentioned by Schaffer: perhaps we do not need to postulate new forces at each level if the levels depend on each other? Be that as it may, we can now draw some preliminary conclusions. At least the following options are open:

1. Boring infinite descent is not physically realizable, for the need to postulate an infinite number of novel forces and exchange particles is not compatible with OWF. Schaffer's symmetric supervenience will not change the matter.

2. Schaffer's requirement of symmetric supervenience for boring infinite descent is too strong. If each level requires novel forces and corresponding exchange particles, then the picture cannot be symmetrical. However, OWF can nevertheless be satisfied, perhaps by something like Dehmelt's model. 
3. Boring infinite descent with symmetric supervenience can be physically realized in accordance with some yet to be specified model that satisfies OWF.

Option (1) is of course undesirable if we hope to show that the combination of metaphysical infinitism and OWF is possible. But Schaffer's requirement of symmetric supervenience is difficult to accommodate in the only provided physical model, whereas the asymmetry of ontological dependence is the received view. This makes option (3) problematic, increasing the attractiveness of option (2), that is, abandoning the symmetry requirement and insisting only on an infinitely repeating structure as a requirement for boring infinite descent. I will not, however, attempt to decide between these options here - the question of the actuality of boring infinite descent remains open.

Finally, it should be acknowledged that the model we have been working with, and hence the preceding discussion, does suffer from one important caveat: no mention has been made of quantum field theory and certain other central aspects of contemporary physics. A worry related to this omission is that the mereological framework for the discussion is unable to accommodate the complications that quantum field theory and the idea of space-time as a continuum introduce. Moreover, physicists have made some, albeit brief, remarks about the topic.

For instance, drawing on issues surrounding renormalization in quantum field theory, Howard Georgi suggests that effective quantum field theories could "go down to arbitrarily short distances in a kind of infinite regression" $(1989,456)$ - a passage also quoted by Schaffer $(2003$, 505). Georgi does not explicitly consider the possibility that the infinite regress could be benign due to being boring, but one promising line of research in this regard might be to apply so-called renormalization group methods developed by Kenneth Wilson and others in the 1970s. ${ }^{8}$ These methods were earlier used by Richard Feynman to treat infinities in quantum field theory in such a way that finite physical quantities can be obtained. The methods can be applied to a number of problems in physics, but in the present context what is interesting is the idea that renormalization group formalism enables the treatment of arbitrarily many (that is, infinitely many) items, such as coupling constants, without breaking down. Specifically, only a finite number of such couplings may be important, so some kind of order of importance can be assigned, which, we might say, renders boring the rest of the items in the infinite series.

Future work on boring infinite descent should certainly take into account these issues, but the purpose of the present article is primarily to

\footnotetext{
${ }^{8}$ See Wilson 1979 or his 1982 Nobel lecture (available at http://www.nobelprize.org/ nobel_prizes/physics/laureates/1982/wilson-lecture.pdf) for a popular introduction to renormalization group methods.
} 
make a start towards an ontological framework for the treatment of boring infinite descent and to defend it as a metaphysically viable model combining metaphysical infinitism and a form of fundamentalism. Regarding the actual world, the issue remains open, as a lot hangs on the physics. What we can conclude with some confidence is that boring infinite descent is at least a possible candidate for maintaining ontological wellfoundedness and hence a form of fundamentalism, while accommodating metaphysical infinitism with some qualifications.

University of Helsinki

Department of Philosophy, History, Culture and Art Studies

P.O. Box 24

00014 University of Helsinki

Finland

tuomas.tahko@helsinki.fi

\section{Acknowledgments}

I would like to thank Einar Bohn, Markku Keinänen, Matteo Morganti, Donnchadh O'Conaill, and Francis "Olley" Pearson for helpful comments on earlier versions of this article. The research for the article was made possible by a grant from the Academy of Finland.

\section{References}

Bennett, K. 2011. "By Our Bootstraps.” Philosophical Perspectives 25:2741.

Bliss, R. L. 2013. "Viciousness and the Structure of Reality." Philosophical Studies 166, no. 2:399-418.

Bohn, E. D. 2009a. "An Argument Against the Necessity of Unrestricted Composition." Analysis 69:27-31.

_ 2009b. "Must There Be a Top Level?" Philosophical Quarterly 59:193-201.

Cameron, R. P. 2008. "Turtles All the Way Down: Regress, Priority and Fundamentality." Philosophical Quarterly 58:1-14.

Correia, F. 2008. "Ontological Dependence." Philosophy Compass 3:1013-32.

Cotnoir, A. J., and A. Bacon. 2012. "Non-wellfounded Mereology." Review of Symbolic Logic 5:187-204.

Dehmelt, H. 1989. "Triton, ... Electron, ... Cosmon, ... : An Infinite Regression?" Proceedings of the National Academy of Sciences 86:861819.

Georgi, H. 1989. "Effective Quantum Field Theories." In The New Physics, edited by Paul Davies, 446-57. Cambridge: Cambridge University Press. 
Kim, J. 2010. Essays in the Metaphysics of Mind. Oxford: Oxford University Press.

Ladyman, J., and D. Ross. 2007. Every Thing Must Go: Metaphysics Naturalized. Oxford: Oxford University Press.

Markosian, N. 2005. "Against Ontological Fundamentalism." Facta Philosophica 7:69-84.

Morganti, M. 2009. "Ontological Priority, Fundamentality and Monism." Dialectica 63, no. 3:271-88.

Paseau, A. 2010. "Defining Ultimate Ontological Basis and the Fundamental Layer." Philosophical Quarterly 60:169-75.

Schaffer, J. 2003. "Is There a Fundamental Level?" Noûs 37:498-517.

- 2009. "On What Grounds What." In Metametaphysics, edited by David Chalmers, David Manley, and Ryan Wasserman, 347-83. Oxford: Oxford University Press.

- 2010. "Monism: The Priority of the Whole." Philosophical Review 119 , no. 1:31-76.

Sider, T. 1993. "Van Inwagen and the Possibility of Gunk." Analysis 53:285-89.

Tallant, J. 2013. "Problems of Parthood for Proponents of Priority." Analysis 73:429-38.

Wilson, K. G. 1979. "Problems in Physics with Many Scales of Length." Scientific American (August): 158-79.

Zimmerman, D. 1996, "Could Extended Objects Be Made Out of Simple Parts? An Argument for 'Atomless Gunk." Philosophy and Phenomenological Research 56:1-29. 